\title{
Efficient Multicomponent Active Template Synthesis of Catenanes
}

\author{
James E. M. Lewis, Florian Modicom, and Stephen M. Goldup*(i) \\ Chemistry, University of Southampton, Highfield, Southampton SO17 1BJ, United Kingdom
}

\section{Supporting Information}

ABSTRACT: We describe a simple and high yielding active template synthesis of [2]catenanes. In addition to mechanical bond formation using a single premacrocycle bearing an azide and alkyne moiety, our method is also suitable for the co-macrocyclization of readily available bisalkyne and bis-azide comonomers and even short alkyne/ azide components which oligomerize prior to mechanical bond formation.

A lthough many of the seminal contributions ${ }^{1}$ to the synthesis of mechanically interlocked molecules ${ }^{2}$ focus on catenanes, ${ }^{3}$ reports of rotaxanes have grown to dominate the field, ${ }^{2 \mathrm{i}}$ in part due to the potential for shuttling motions in rotaxanes that makes them attractive for the development of molecular machines. ${ }^{4,5}$ However, the higher synthetic challenge involved in catenane synthesis probably plays a role; whereas rotaxanes can be synthesized readily using threading-thenstoppering methodologies, catenane synthesis requires a macrocyclization event to capture the interlocked architecture with the attendant competition between cyclization and oligomerization. To overcome this, catenanes are typically formed under high-dilution conditions, leading to long reaction times and, where the association between the preformed ring and the macrocycle precursor is weak, diminished yields.

Despite these challenges a variety of catenane syntheses have been disclosed, each with their own advantages and disadvantages. ${ }^{2}$ Reactions that allow the assembly of the new macrocycle from small building blocks such as Stoddart's donor-acceptor ${ }^{6}$ and pimer $^{7}$ systems, and the amide-templated examples developed by Vögtle, Hunter and Leigh are particularly attractive due to their synthetic efficiency. ${ }^{8}$ Similarly, metal-directed passive template (PT) approaches, exemplified by Sauvage's $\mathrm{Cu}^{\mathrm{I}}$-phenanthroline methodology, are widely used thanks to the strong metal-ligand interactions that favor association of the components. ${ }^{9}$ However, these strategies also exemplify the drawbacks of many current methodologies. The former rely on preorganization of the incipient macrocycle through interactions that simultaneously favor cyclization and catenane formation. This leads to high yields in ideal cases, but minimal structural changes can disrupt these interactions, significantly lowering the yield. ${ }^{8 \mathrm{e}}$ Conversely, metal-directed PT approaches require high-dilution conditions to prevent oligomerization and, although the stability of the precursor complex ensures efficient threading, cyclization is typically slow. PT approaches are also ill-suited to the synthesis of sterically hindered products as repulsive interactions disfavor threaded complex formation.
The active template (AT) approach to rotaxanes ${ }^{10}$ has the potential to overcome many of the outstanding challenges in catenane synthesis as AT-reactions typically proceed in high yield and are extremely general with respect to the substrates employed, including sterically hindered examples. ${ }^{11}$ However, although Leigh and Saito have disclosed AT catenane syntheses, ${ }^{12}$ they proceed at low concentration over long reaction times and require a large excess of macrocycle precursor to achieve reasonable yields.

We recently optimized our small macrocycle ${ }^{13}$ modification of Leigh's AT Cu-mediated azide-alkyne cycloaddition (ATCuAAC $)^{14}$ reaction for the rapid and efficient iterative synthesis of oligorotaxanes. ${ }^{15}$ This suggested an opportunity as, were these advantages to be maintained in the formation of catenanes, these conditions might allow efficient pseudo-high dilution reactions. Here we report that this approach is extremely successful, producing catenanes in good to excellent yield with short reaction times. The reaction is general with respect to both macrocycle and premacrocycle structure, allowing the synthesis of small, crowded catenanes and the use of simple building blocks via a controlled oligomerization pathway.

Precursor 1 was readily prepared and its reaction with macrocycle $2 a^{11}$ investigated with respect to solvent, temperature, concentration and rate of addition of $\mathbf{1}$ to $\mathbf{2 a}$, to identify the variables that control reaction selectivity. Unsurprisingly, catenane formation was favored when the addition time, temperature and concentration of $\mathbf{1}$ and $\mathbf{2 a}$ were balanced to maintain a low instantaneous concentration of 2a (see ESI). Using this information, we designed general conditions for the synthesis of catenanes 3 from macrocycles $\mathbf{2}$ (Scheme 1 ). When 1 equiv of $\mathbf{1}$ was added to macrocycle $2 \mathrm{a}$ in the presence of $\left[\mathrm{Cu}(\mathrm{MeCN})_{4}\right] \mathrm{PF}_{6}$ and ${ }^{i} \mathrm{Pr}_{2} \mathrm{NEt}$ over $4 \mathrm{~h}, 2 \mathrm{2a}$ was completely consumed and catenane 3a was isolated in 94\% yield. Larger macrocycle $\mathbf{2} \mathbf{b}$ was $88 \%$ converted to catenane $\mathbf{3 b}$ on addition of 1 equiv of 1 . Variation of the addition rate or reaction temperature did not alter the reaction selectivity, indicating that macrocycle $3 \mathbf{a}$ has an inherently lower selectivity for mechanical bond formation. When 1.5 equiv of 1 (entry 2) were used, $\mathbf{2} \mathbf{b}$ was completely consumed and $\mathbf{3 b}$ was isolated in $96 \%$ yield. Macrocycle $2 \mathrm{c}$ also required modification of the conditions to overcome a slower reaction and diminished selectivity for the catenane compared with $2 \mathrm{a}$; addition of 1.5 equiv of 1 to $2 \mathrm{c}$ at $80{ }^{\circ} \mathrm{C}$ allowed quantitative consumption of $2 \mathrm{c}$ and isolation of [2]catenane $3 \mathrm{c}$ in $98 \%$ yield (entry 3 ). Macrocycle $2 \mathbf{d}$ required elevated temperatures to achieve $\sim 94 \%$ conversion to catenane $3 \mathrm{~d}$. Coelution of the $3 \mathrm{~d}$ with the

Received: February 8, 2018

Published: March 20, 2018 
Scheme 1. Efficient AT-CuAAC Syntheses of [2]Catenanes $3^{a, b, c, d}$

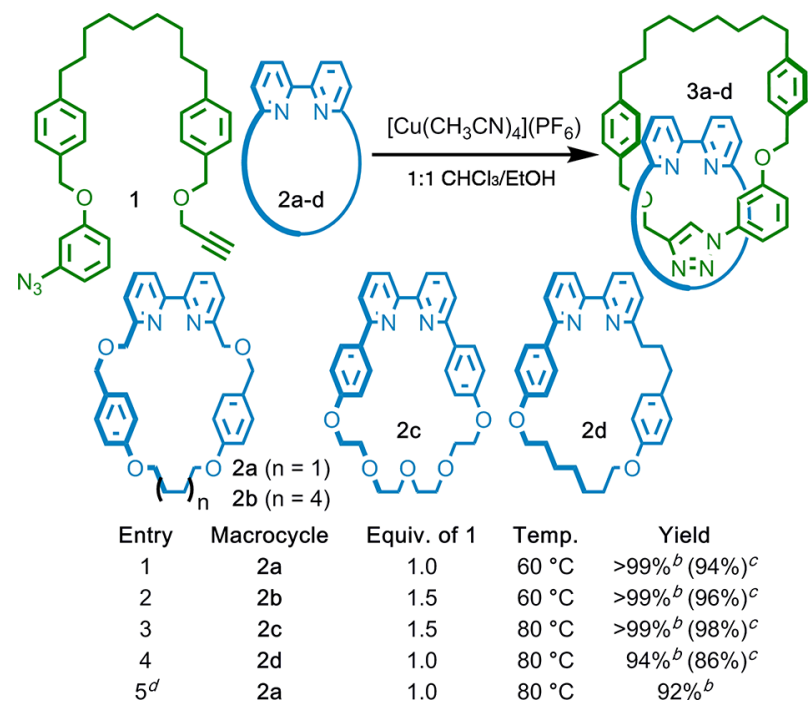

${ }^{a}$ Reagents and conditions: $\mathbf{1}$ in 1:1 $\mathrm{CHCl}_{3}-\mathrm{EtOH}(25 \mathrm{mM})$ added to $2(25 \mathrm{mM}),{ }^{i} \mathrm{Pr}_{2} \mathrm{NEt}$ (2 equiv) and $\left[\mathrm{Cu}\left(\mathrm{CH}_{3} \mathrm{CN}\right)_{4}\right]\left(\mathrm{PF}_{6}\right)$ (0.99 equiv) in $1: 1 \mathrm{CHCl}_{3}-\mathrm{EtOH}$ over $4 \mathrm{~h}$. ${ }^{b}$ Determined by ${ }^{1} \mathrm{H}$ NMR analysis. ${ }^{c}$ Isolated yield. ${ }^{d}$ Concentration of $\mathbf{1} / \mathbf{2 a}=100 \mathrm{mM}$.

macrocycle derived from 1 precluded addition of excess 1 to increase the conversion and resulted in a diminished isolated yield of $86 \%$ (entry 4). Although the reactions of macrocycles 2 with $\mathbf{1}$ are all extremely efficient, ${ }^{16}$ the reaction of $\mathbf{2 a}$ with $\mathbf{1}$ is particularly robust; 3a was produced in excellent yield (92\%) even when the concentration of $2 \mathrm{a}$ was increased to $100 \mathrm{mM}$ (entry 5).

The high selectivity of the reactions to produce catenanes 3 suggested that the AT-CuAAC reaction would be suitable for a multicomponent cyclization approach to catenanes from small, simple starting materials by a controlled oligomerization pathway, a significant benefit for the production of interlocked molecules on larger scales. To this end, bis-alkyne and bis-azide containing precursors were prepared and reacted with macrocycle $\mathbf{2} \mathbf{a}$ under our standard conditions and the reactions briefly optimized with respect to equivalents of precursors and addition time. Using this approach, catenanes 4 and 5 were produced in good isolated yield through the cocyclization of a diazide and dipropargyl ether or 2,6-diethynylpyridine, respectively (Figure 1). Analysis of the crude reaction mixtures indicated that the smallest possible [2]catenanes were produced with complete selectivity (Figures S139-40). Our multicomponent synthesis is also suitable for the reaction of $\alpha, \alpha^{\prime}$-diazido- $p$-xylene with either dipropargyl ether or 2,6diethynylpyridine to produce catenanes 6 and 7 in isolated yields of $78 \%$ and $72 \%$ respectively. In these reactions, the smallest triazole-containing macrocycle that can lead a [2] catenane requires the formation of a heterotetramer as the corresponding heterodimeric macrocycle is too small to encircle 2a. Once again, the hetereo-tetrameric species was the only observed interlocked product (Figures S141-2).

Having successfully demonstrated a hetero-oligomerizationthen-cyclization approach to [2]catenanes, we turned our attention to the equivalent homocyclization process. Using an azide-alkyne in which the reactive functional groups are 11 atoms apart led to catenane $\mathbf{8}$ in which the triazole-containing 28 membered ring is homodimeric in $84 \%$ yield. In this case,

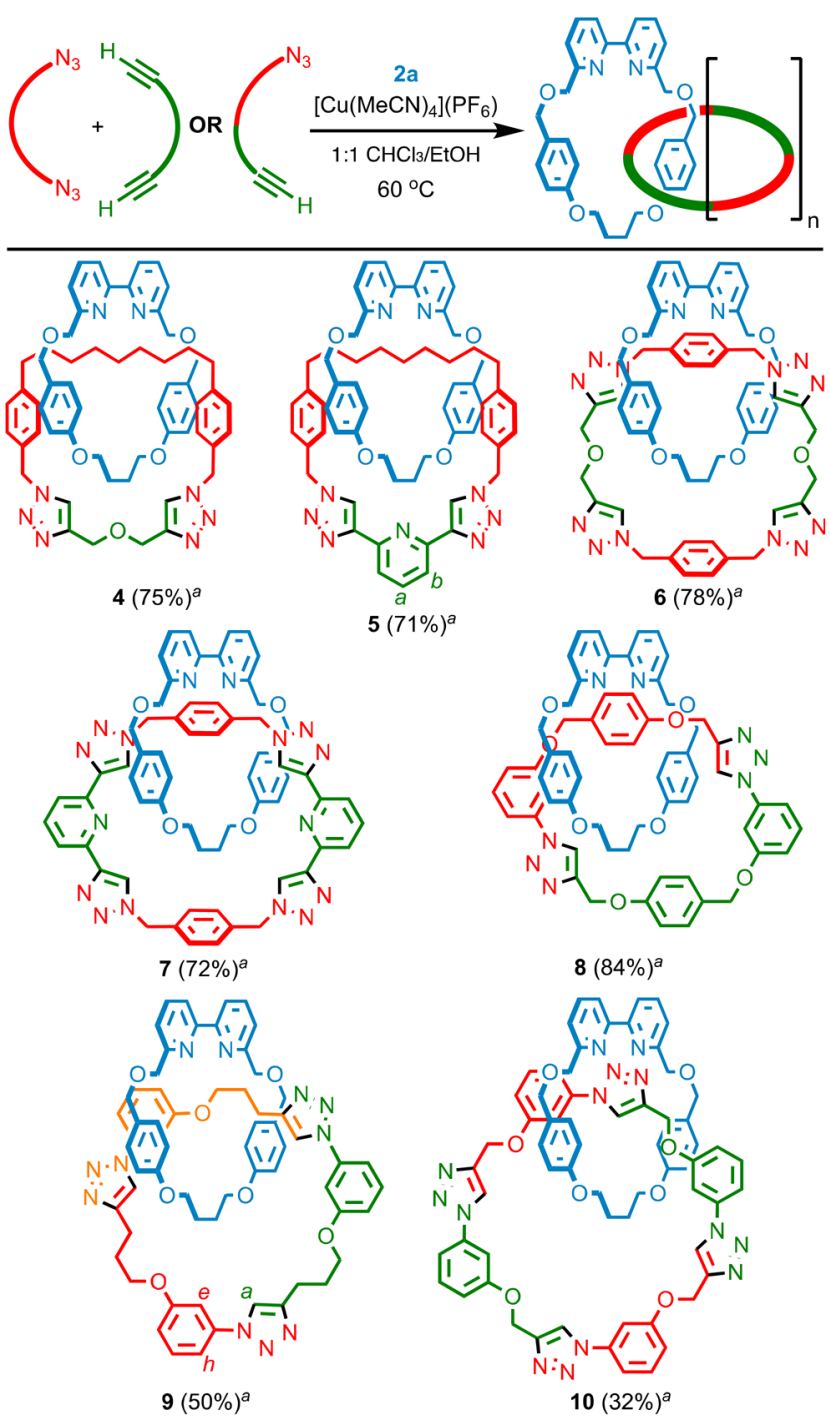

Figure 1. Multicomponent AT-CuAAC [2]Catenanes 4-10. ${ }^{a}$ Isolated yield. ${ }^{17}$

$\sim 10 \%$ of the corresponding homotrimeric catenane was also observed (Figure S143). Decreasing the distance between the alkyne and azide functional groups to 7 atoms resulted in a $50 \%$ yield of catenane $\mathbf{9}^{17}$ in which the 30 membered triazolecontaining ring is homotrimeric. Both the corresponding homotetramer- and homopentamer-derived catenanes were also observed in a combined ratio of $\sim 1: 2$ with 9 (Figure S144). Finally, using a precursor in which the reactive functionality are 5 atoms apart resulted in a $32 \%$ isolated yield of tetramer-derived catenane $\mathbf{1 0}$. In this case, a range of impurities were observed up to and including the homoheptamer-derived product in a combined $\sim 1: 2$ ratio with 10 (Figure S145). Thus, although effective, the homo-oligomerization-then-cyclization approach appears to be less selective for a single interlocked product than the heterocyclization pathway.

Catenanes 3-10 were fully characterized by NMR and MS (see ESI). In addition, catenanes $3 \mathbf{b}, 3 \mathbf{d}, \mathbf{5}, 7$, and 9 were characterized by single crystal X-ray diffraction (Figures S146S150). Their solid-state structures suggest that, absent the significant stabilizing intercomponent interactions typically found in interlocked molecules produced using PT approaches, the rings adopt coconformations that maximize weak, 
stabilizing contacts such as $\mathrm{C}-\mathrm{H}$ H-bonding, $\mathrm{C}-\mathrm{H}-\pi$ and $\pi-\pi$ interactions due to the enforced proximity of the covalent subcomponents (e.g., Figure 2a). In keeping with this, longer contacts were observed in catenane $\mathbf{3 b}$ which contains larger, more flexible bipyridine macrocycle $\mathbf{2} \mathbf{b}$.
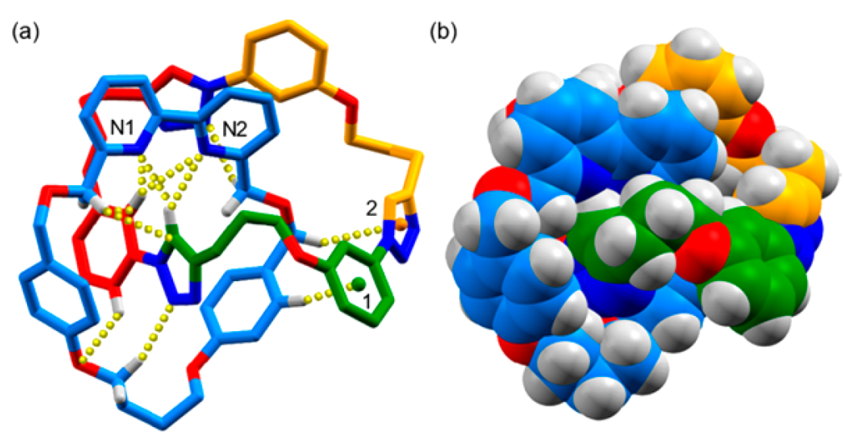

Figure 2. Solid-state structure of catenane 9 in (a) tube and (b) spacefilling representations. Selected intercomponent distances in $\AA$ : $\mathrm{H}_{a}-\mathrm{N}_{1}=2.34, \mathrm{H}_{a}-\mathrm{N}_{2}=2.76 ; \mathrm{H}_{a}-\mathrm{N}_{1}=2.75, \mathrm{H}_{a}-\mathrm{N}_{2}=2.77 ; \mathrm{H}_{h}-\mathrm{O}=$ 2.86; $\mathrm{H}_{D}-\mathrm{C}_{a}=2.94 ; \mathrm{H}_{D}-\mathrm{C}_{e}=2.96 ; \mathrm{H}_{D^{\prime}}-\mathrm{N}=2.99 ; \mathrm{H}_{E-\bullet 2}=2.83$; $\mathrm{H}_{F-\bullet 1}=2.81 ; \mathrm{H}_{H}-\mathrm{N}=2.44$. Atom labels as in Figure 1 .

The solid-state structures of $\mathbf{3 b}, \mathbf{3 d}, \mathbf{5}, \mathbf{7}$ and $\mathbf{9}$ all contain a racemic mixture of enantiomers that are related by points of inversion (3b, 3d, 5, and 7 ) or glide planes (9). This is expected in the case of $3 \mathbf{d}$, which is composed of two $C_{s^{-}}$. symmetric macrocycles and is thus topologically chiral. ${ }^{18}$ However, although $\mathbf{3 b}, \mathbf{5}, \mathbf{7}$, and 9 are expected to be, on average, achiral as they contain at least one $C_{2 \mathrm{v}}$-symmetric macrocycle, the relative arrangements of the two ring components in the solid-state produces enantiomeric coconformations in which each macrocycle desymmetrizes the other, leading to structures with similar stereochemical properties to catenane 3d. This coconformational stereochemistry is similar to [2] rotaxanes in which the relative arrangement of the macrocycle and a $C_{2 \mathrm{v}}$ or $C_{\mathrm{s}}$ axle leads to mechanical planar ${ }^{19}$ or covalent point ${ }^{20}$ stereogenic elements, respectively. However, to our knowledge the coconformational chiral stereogenic element of catenanes $3 \mathbf{b}, \mathbf{5}, \mathbf{7}$, and 9 has not been highlighted previously. However, despite their crowded nature and the interactions and stereochemistry observed in the solid state, the ${ }^{1} \mathrm{H}$ NMR spectra of catenanes 3-10 are highly symmetrical with only one triazole resonance observed, suggesting they remain mobile on the ${ }^{1} \mathrm{H}$ NMR time scale in solution. ${ }^{2}$

The yield of the reactions presented is particularly striking as selectivity for both mechanical bond formation and macrocyclization over uncontrolled oligomerization is required for efficient catenane synthesis. Our original hypothesis was that this could be achieved by optimizing the bipyridine-mediated AT-CuAAC reaction, which is highly selective for interlocked products $^{10 \mathrm{~b}, 11,13,15}$ as the alkyne and azide reactive functional groups are projected on opposite faces of the ring in the key bond forming step, ${ }^{28}$ to proceed rapidly under pseudo-high dilution conditions in order to maintain a low instantaneous concentration of the substrates. However, it remained unclear whether secondary interactions between the bipyridine macrocycle and the nascent triazole macrocycle also played a significant role in preorganizing the reaction intermediates to lead to a single major catenane product, although the broad substrate scope observed suggested this was not the case. To investigate this, the reaction of $\mathbf{1}$ was performed in the absence of a bipyridine ligand. Analysis of the crude reaction mixture by ${ }^{1} \mathrm{H}$ NMR and LCMS (Figures S133, S138) revealed selective formation of the corresponding monomeric triazole macrocycle, consistent with a simple pseudo-high dilution macrocyclization process. Thus, it seems that the origin of the high yield of our methodology is a combination of the selectivity of the AT-CuAAC reaction and a rapid macrocyclization process, leading to the catenane containing the smallest possible triazole-containing macrocycle. Importantly, the simplicity of this model allows the rational optimization of conditions for different substrates by balancing the rate of addition and the reaction rate.

In conclusion, we have developed a facile, high-yielding and general AT-CuAAC approach for the synthesis of [2]catenanes that allows a multicomponent approach in which readily available precursors, too small to cyclize directly, undergo successive reactions until a final AT-CuAAC reaction gives the interlocked target. Furthermore, the products reported here contain unusually small macrocycles of between 26 and 32 atoms and viewing their structures in space-filling representation (e.g., Figure $2 \mathrm{~b}$ ) serves to underline the crowded nature of the mechanical bond which belies the high yield of their formation. Indeed, catenane 3a contains rings that are 26 and 28 atoms in circumference, and yet it forms in near quantitative yield. In contrast, Sauvage and co-workers found that otherwise identical catenanes were formed in $42 \%{ }^{1 \mathrm{~d}}$ or $3.3 \%{ }^{22}$ yield when the macrocycles to be interlocked were 30 or 27 atoms in circumference respectively, demonstrating the sensitivity of the PT approach to destabilizing steric interactions. Despite the historical significance of catenanes ${ }^{1,3}$ and although existing methodologies have allowed ever more complex targets to be realized, ${ }^{23}$ the relative challenge of catenane synthesis compared to rotaxanes has contributed to making them less well examined targets. With this new flexible methodology in hand, AT-CuAAC catenanes are as easy if not easier to access than their rotaxane counterparts. Given the wide range of applications for AT-CuAAC-derived rotaxanes, including as catalysts, ${ }^{24}$ ligands, ${ }^{25}$ hosts/sensors, ${ }^{26}$ and molecular machines, ${ }^{27}$ and the AT-CuAAC reaction's proven ability to access complex threaded systems efficiently, ${ }^{28}$ we anticipate a similar surge in applications of AT-CuAAC catenanes.

\section{ASSOCIATED CONTENT}

\section{Supporting Information}

The Supporting Information is available free of charge on the ACS Publications website at DOI: 10.1021/jacs.8b01602.

Full synthetic details and characterization data (PDF)

$\mathrm{X}$-ray data for compound $\mathbf{3 b}$ (CIF)

$\mathrm{X}$-ray data for compound $\mathbf{3 d}$ (CIF)

$\mathrm{X}$-ray data for compound 5 (CIF)

$\mathrm{X}$-ray data for compound 7 (CIF)

$\mathrm{X}$-ray data for compound 9 (CIF)

\section{AUTHOR INFORMATION}

\section{Corresponding Author}

*s.goldup@soton.ac.uk

ORCID $\odot$

Stephen M. Goldup: 0000-0003-3781-0464

Notes

The authors declare no competing financial interest. 


\section{ACKNOWLEDGMENTS}

The authors thank Fluorochem for the gift of reagents. J.E.M.L. thanks the European Union's Horizon 2020 programme for a Marie Skłodowska-Curie Fellowship (agreement no. 660731). F.M. thanks the EPSRC (EP/L505067/1) for funding. S.M.G. acknowledges funding from the European Research Council (Consolidator Grant Agreement no. 724987).

\section{REFERENCES}

(1) (a) Wasserman, E. J. Am. Chem. Soc. 1960, 82, 4433. (b) Wasserman, E. J. Am. Chem. Soc. 1960, 82, 4433. (c) Schill, G.; Lüttringhaus, A. Angew. Chem., Int. Ed. Engl. 1964, 3, 546. (d) DietrichBuchecker, C. O.; Sauvage, J. P.; Kintzinger, J. P. Tetrahedron Lett. 1983, 24, 5095. (e) Ashton, P. R.; Goodnow, T. T.; Kaifer, A. E.; Reddington, M. V.; Slawin, A. M. Z.; Spencer, N.; Stoddart, J. F.; Vicent, C.; Williams, D. J. Angew. Chem., Int. Ed. Engl. 1989, 28, 1396.

(2) Reviews on the synthesis and applications of interlocked molecules: (a) Vickers, M. S.; Beer, P. D. Chem. Soc. Rev. 2007, 36, 211. (b) Stoddart, J. F. Chem. Soc. Rev. 2009, 38, 1802. (c) Beves, J. E.; Blight, B. A.; Campbell, C. J.; Leigh, D. A.; McBurney, R. T. Angew. Chem., Int. Ed. 2011, 50, 9260. (d) Neal, E. A.; Goldup, S. M. Chem. Commun. 2014, 50, 5128. (f) Erbas-Cakmak, S.; Leigh, D. A.; McTernan, C. T.; Nussbaumer, A. L. Chem. Rev. 2015, 115, 10081. (g) Xue, M.; Yang, Y.; Chi, X.; Yan, X.; Huang, F. Chem. Rev. 2015, 115, 7398. (h) Lewis, J. E. M.; Galli, M.; Goldup, S. M. Chem. Commun. 2017, 53, 298. (i) Bruns, C. J.; Stoddart, J. F. The Nature of the Mechanical Bond; John Wiley \& Sons, Inc.: Hoboken, NJ, USA, 2016. (j) Lewis, J. E. M.; Beer, P. D.; Loeb, S. J.; Goldup, S. M. Chem. Soc. Rev. 2017, 46, 2577.

(3) Reviews that focus on catenanes: (a) Evans, N. H.; Beer, P. D. Chem. Soc. Rev. 2014, 43, 4658. (b) Gil-Ramírez, G.; Leigh, D. A.; Stephens, A. J. Angew. Chem., Int. Ed. 2015, 54, 6110.

(4) Reviews on the development of molecular machines: (a) Balzani, V.; Credi, A.; Venturi, M. Chem. Soc. Rev. 2009, 38, 1542. (b) Coskun, A.; Banaszak, M.; Astumian, R. D.; Stoddart, J. F.; Grzybowski, B. A. Chem. Soc. Rev. 2012, 41, 19. (c) Erbas-Cakmak, S.; Leigh, D. A.; McTernan, C. T.; Nussbaumer, A. L. Chem. Rev. 2015, 115, 10081. (d) Leigh, D. A. Angew. Chem., Int. Ed. 2016, 55, 14506.

(5) Nobel lectures on the subject of molecular machines: (a) Sauvage, J. P. Angew. Chem., Int. Ed. 2017, 56, 11080. (b) Stoddart, J. F. Angew. Chem., Int. Ed. 2017, 56, 11094. (c) Feringa, B. L. Angew. Chem., Int. Ed. 2017, 56, 11060.

(6) See ref le and: (a) Fahrenbach, A. C.; Bruns, C. J.; Cao, D.; Stoddart, J. F. Acc. Chem. Res. 2012, 45, 1581. (b) Fahrenbach, A. C.; Bruns, C. J.; Li, H.; Trabolsi, A.; Coskun, A.; Stoddart, J. F. Acc. Chem. Res. 2014, 47, 482.

(7) Barnes, J. C.; Fahrenbach, A. C.; Cao, D.; Dyar, S. M.; Frasconi, M.; Giesener, M. A.; Benítez, D.; Tkatchouk, E.; Chernyashevskyy, O.; Shin, W. H.; Li, H.; Sampath, S.; Stern, C. L.; Sarjeant, A. A.; Hartlieb, K. J.; Liu, Z.; Carmieli, R.; Botros, Y. Y.; Choi, J. W.; Slawin, A. M. Z.; Ketterson, J. B.; Wasielewski, M. R.; Goddard, W. A., III; Stoddart, J. F. Science 2013, 339, 429.

(8) (a) Vögtle, F.; Meier, S.; Hoss, R. Angew. Chem., Int. Ed. Engl. 1992, 31, 1619. (b) Hunter, C. A. J. Am. Chem. Soc. 1992, 114, 5303. (c) Johnston, A. G.; Leigh, D. A.; Pritchard, R. J.; Deegan, M. D. Angew. Chem., Int. Ed. Engl. 1995, 34, 1209. (d) Vögtle, F.; Dünnwald, T.; Schmidt, T. Acc. Chem. Res. 1996, 29, 451. (e) Leigh, D. A.; Venturini, A.; Wilson, A. J.; Wong, J. K. Y.; Zerbetto, F. Chem. - Eur. J. 2004, 10, 4960 .

(9) (a) Dietrich-Buchecker, C. O.; Sauvage, J. P.; Kintzinger, J. Tetrahedron Lett. 1983, 24 (46), 5095. (b) Dietrich-Buchecker, C. O.; Sauvage, J. P.; Kern, J. M. J. Am. Chem. Soc. 1984, 106, 3043.

(10) (a) Crowley, J. D.; Goldup, S. M.; Lee, A.-L.; Leigh, D. A.; McBurney, R. T. Chem. Soc. Rev. 2009, 38, 1530. (b) Denis, M.; Goldup, S. M. Nat. Rev. Chem. 2017, 1, 61.

(11) Lewis, J. E. M.; Bordoli, R. J.; Denis, M.; Fletcher, C. J.; Galli, M.; Neal, E. A.; Rochette, E. M.; Goldup, S. M. Chem. Sci. 2016, 7, 3154.
(12) (a) Sato, Y.; Yamasaki, R.; Saito, S. Angew. Chem., Int. Ed. 2009, 48, 504. (b) Goldup, S. M.; Leigh, D. A.; Long, T.; McGonigal, P. R.; Symes, M. D.; Wu, J. J. Am. Chem. Soc. 2009, 131, 15924. (c) Ito, K.; Mutoh, Y.; Saito, S. J. Org. Chem. 2017, 82, 6118.

(13) Lahlali, H.; Jobe, K.; Watkinson, M.; Goldup, S. M. Angew. Chem., Int. Ed. 2011, 50, 4151.

(14) (a) Aucagne, V.; Hänni, K. D.; Leigh, D. A.; Lusby, P. J.; Walker, D. B. J. Am. Chem. Soc. 2006, 128, 2186. (b) Aucagne, V.; Berna, J.; Crowley, J. D.; Goldup, S. M.; Hänni, K. D.; Leigh, D. A.; Lusby, P. J.; Ronaldson, V. E.; Slawin, A. M. Z.; Viterisi, A.; Walker, D. B. J. Am. Chem. Soc. 2007, 129, 11950.

(15) (a) Lewis, J. E. M.; Winn, J.; Cera, L.; Goldup, S. M. J. Am. Chem. Soc. 2016, 138, 16329. (b) Lewis, J. E. M.; Winn, J.; Goldup, S. M. Molecules 2017, 22, 89.

(16) $\mathbf{2 a}$ and $\mathbf{2 b}$ produce $3 \mathbf{a}$ and $\mathbf{3 b}$ respectively as the sole interlocked products (Figures S134-5). The crude reaction mixtures of $2 \mathrm{c}$ and $2 \mathrm{~d}$ contained traces of [2]catenanes derived from the homodimer of 1 (Figure S136-7).

(17) The isolated yield of 9 was determined from the mass of a purified sample contaminated with $\sim 20 \%$ dimeric triazole macrocycle S14 (see ESI). A small quantity of analytically pure of 9 was obtained by crystallization for characterization.

(18) For selected recent reviews that discuss chirality in interlocked molecules see ref 2d,i and: (a) Evans, N. H. Chem. - Eur. J. 2018, 24 3101. (b) Pairault, N.; Niemeyer, J. Synlett 2018, 29, 689.

(19) Mochizuki, Y.; Ikeyatsu, K.; Mutoh, Y.; Hosoya, S.; Saito, S. Org Lett. 2017, 19, 4347.

(20) (a) Alvarez-Pérez, M.; Goldup, S. M.; Leigh, D. A.; Slawin, A. M. Z. J. Am. Chem. Soc. 2008, 130, 1836. (b) Carlone, A.; Goldup, S. M.; Lebrasseur, N.; Leigh, D. A.; Wilson, A. J. Am. Chem. Soc. 2012, 134, 8321.

(21) $\mathrm{H}_{a}$ of 5 displays a double-doublet splitting by ${ }^{1} \mathrm{H}$ NMR suggesting that $\mathrm{H}_{b}$ are inequivalent. 5 and 7 also exhibit broadening of other signals which may indicate that pirouetting is slower in these cases.

(22) Dietrich-Buchecker, C. O.; Edel, A.; Kintzinger, J. P.; Sauvage, J. P. Tetrahedron 1987, 43, 333.

(23) Selected examples: (a) Amabilino, D. B.; Ashton, P. R.; Reder, A. S.; Spencer, N.; Stoddart, J. F. Angew. Chem., Int. Ed. Engl. 1994, 33, 1286. (b) Leigh, D. A.; Pritchard, R. G.; Stephens, A. J. Nat. Chem. 2014, 6, 978. (c) Wood, C. S.; Ronson, T. K.; Belenguer, A. M.; Holstein, J. J.; Nitschke, J. R. Nat. Chem. 2015, 7, 354. (d) Zhu, R.; Lübben, J.; Dittrich, B.; Clever, G. H. Angew. Chem., Int. Ed. 2015, 54, 2796. (e) Black, S. P.; Wood, D. M.; Schwarz, F. B.; Ronson, T. K.; Holstein, J. J.; Stefankiewicz, A. R.; Schalley, C. A.; Sanders, J. K. M.; Nitschke, J. R. Chem. Sci. 2016, 7, 2614. (f) Sawada, T.; Yamagami, M.; Ohara, K.; Yamaguchi, K.; Fujita, M. Angew. Chem., Int. Ed. 2016, 55, 4519.

(24) (a) Hoekman, S.; Kitching, M. O.; Leigh, D. A.; Papmeyer, M.; Roke, D. J. Am. Chem. Soc. 2015, 137 (24), 7656. (b) Galli, M.; Lewis, J. E. M.; Goldup, S. M. Angew. Chem., Int. Ed. 2015, 54, 13545.

(25) (a) Winn, J.; Pinczewska, A.; Goldup, S. M. J. Am. Chem. Soc. 2013, 135 (36), 13318. (b) Noor, A.; Lo, W. K. C.; Moratti, S. C.; Crowley, J. D. Chem. Commun. 2014, 50, 7044. (c) Noor, A.; Moratti, S. C.; Crowley, J. D. Chem. Sci. 2014, 5, 4283. (d) Ngo, T. H.; Labuta, J.; Lim, G. N.; Webre, W.; D'Souza, F.; Karr, P. A.; Lewis, J. E. M.; Hill, J. P.; Ariga, K.; Goldup, S. Chem. Sci. 2017, 8, 6679.

(26) (a) Langton, M. J.; Xiong, Y.; Beer, P. D. Chem. - Eur. J. 2015, 21, 18910. (b) Brown, A.; Lang, T.; Mullen, K. M.; Beer, P. D. Org. Biomol. Chem. 2017, 15, 4587. (c) Lim, J. Y. C.; Bunchuay, T.; Beer, P. D. Chem. - Eur. J. 2017, 23, 4700. (d) Lim, J. Y. C.; Marques, I.; Thompson, A. L.; Christensen, K. E.; Félix, V.; Beer, P. D. J. Am. Chem. Soc. 2017, 139, 3122. (e) Denis, M.; Qin, L.; Turner, P.; Jolliffe, K.; Goldup, S. M. Angew. Chem., Int. Ed. 2018, DOI: 10.1002/ anie.201713105. (f) Denis, M.; Pancholi, J.; Jobe, K.; Watkinson, M.; Goldup, S. M. Angew. Chem., Int. Ed. 2018, DOI: 10.1002/ anie.201712931.

(27) See ref 11 and: (a) Lewandowski, B.; De Bo, G.; Ward, J. W.; Papmeyer, M.; Kuschel, S.; Aldegunde, M. J.; Gramlich, P. M. E.; 
Heckmann, D.; Goldup, S. M.; D’Souza, D. M.; Fernandes, A. E.; Leigh, D. A. Science 2013, 339, 189. (b) De Bo, G.; Kuschel, S.; Leigh, D. a; Lewandowski, B.; Papmeyer, M.; Ward, J. W. J. Am. Chem. Soc. 2014, 136, 5811. (c) De Bo, G.; Gall, M. A. Y.; Kitching, M. O.; Kuschel, S.; Leigh, D. A.; Tetlow, D. J.; Ward, J. W. J. Am. Chem. Soc. 2017, 139, 10875 .

(28) See 14b and: (a) Goldup, S. M.; Leigh, D. A.; McGonigal, P. R.; Ronaldson, V. E.; Slawin, A. M. Z. J. Am. Chem. Soc. 2010, 132, 315.

(b) Barran, P. E.; Cole, H. L.; Goldup, S. M.; Leigh, D. A.; McGonigal, P. R.; Symes, M. D.; Wu, J.; Zengerle, M. Angew. Chem., Int. Ed. 2011, 50, 12280. (c) Bordoli, R. J.; Goldup, S. M. J. Am. Chem. Soc. 2014, 136, 4817. (e) Neal, E. A.; Goldup, S. M. Chem. Sci. 2015, 6, 2398.

(f) Neal, E. A.; Goldup, S. M. Angew. Chem., Int. Ed. 2016, 55, 12488. 